\title{
Investigation of turbulence rotation and radial electric field in the island divertor and plasma edge at W7-X
}

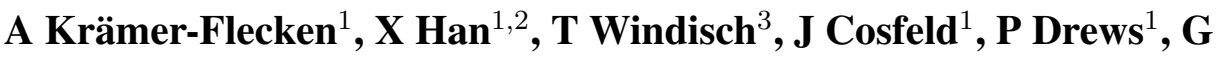

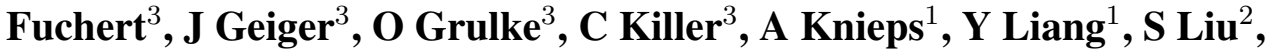 \\ $M$ Rack $^{1}$ and the W7-X team $\ddagger$ \\ 1 Institute for Climate and Energy Research - Plasma Physics, Forschungszentrum Jülich, \\ 52425 Jülich, Germany \\ 2 Institute of Plasma Physics, Chinese Academy of Sciences, Hefei 230031, People's \\ Republic of China \\ 3 Max Planck Institut für Plasmaphysik, 17491 Greifswald, Germany \\ E-mail: a.kraemer-fleckenefz-juelich.de
}

\begin{abstract}
Diverted plasmas in the stellarator W7-X are investigated using a hopping Poloidal Correlation Reflectometer and a set of Langmuir probes to investigate the plasma edge and the scrape of layer at two different toroidal positions and poloidal cross sections, respectively.

The properties of the scrape off layer are studied as function of two magnetic configurations and as function of plasma parameters. The experimentally determined radial position of the inversion point of the radial electric field is compared with calculations. For certain magnetic configurations a remnant island in the scrape of layer is observed and studied as function of the plasma current.

Different methods to determine the radial electric field are applied which allow to estimate the phase velocity of the turbulence. The measured phase velocity is small and shows some evidence for the existence of resistive ballooning modes in the scrape of layer.

Spectrum decomposition of coherence spectra is applied for a radial localization of modes and to determine their properties. An estimate of the radial correlation length of the broad band turbulence in the vicinity of the last closed flux surface is calculated from the decrease of broad band turbulence amplitude as obtained from the coherence spectra. The obtained radial correlation length is found to be $\lambda_{r} \approx 15 \mathrm{~mm}$
\end{abstract}

Keywords: stellarator, correlation reflectometer, turbulence rotation, radial electric field, scrape off layer

Submitted to: Plasma Phys. Control. Fusion

$\ddagger$ Author list Nuclear Fusion Vol. 53 No. 12 Article 126001 (2013) 


\section{Introduction}

The superconducting stellarator W7-X [1, 2] has a large flexibility in the magnetic configuration. As a consequence island chains with different mode numbers are generated at the plasma edge and in the scrape off layer (SOL) resulting in an island divertor geometry [3]. Additional trim coils are available for error field correction and control coils to manipulate the size and the poloidal location of the edge island chains.

The paper focuses on the measurement of radial profiles of turbulence rotation $\left(v_{\perp}\right)$ and radial electric field $\left(E_{r}\right)$ in two magnetic configurations and for different plasma conditions. The experimentally observed inversion point of $E_{r}$ is a measure for the radial position of the last closed flux surface (LCFS). This position is compared with VMEC calculations of the LCFS and compared to the position where a steep increase of the field line connection length $\left(L_{c}\right)$ is observed. From the difference of the turbulence- and the $E \times B$-rotation the nature of the underlying instability can be determined. This difference can be accessed from the measurement of $E_{r}$ from two different diagnostics and methods, respectively (i) from the turbulence propagation time calculation from poloidal correlation reflectometry and (ii) directly from the measurement of the floating potential.

Due to a self generated plasma current [4] the preset magnetic configuration is perturbed and influences the size of the island chain in the SOL. The effect of this plasma current on the turbulence rotation measurement in the SOL will be studied.

The spectral behaviour of quasi coherent modes and broad band turbulence is monitored in the plasma edge and the LCFS. Due to the strong shear layer at the LCFS tearing apart the eddies with low wave number it is possible to estimate the radial correlation length of the broad band turbulence from the amplitude evolution of this mode.

The paper is organized as follows: Section 2 describes the used diagnostic and the measurement. Section 3 will discuss the observations in the plasma edge and SOL with respect to measured profiles and turbulence properties. Section 4 will summarize and conclude the measurements.

\section{Diagnostics and measurement}

The paper discusses two different magnetic configurations. The first one is the standard configuration denoted in the paper as EJM which is the ideal configuration plus additional trim coils to compensate the $m=1$ error field. The standard configuration has an $\iota \approx 1$ at the LCFS and a large 5/5-island chain is dominating in the SOL. The second configuration investigated is the high iota- configuration denoted as FTM with $\iota \approx 1.2$ at the plasma edge. The two configurations are expected to be quite different in the width and structure of the SOL.

At W7-X a Poloidal Correlation Reflectometer (PCR) has been installed and operated in the first two experimental campaigns. The system is described in refs $[5,6]$. It operates in O-mode polarization in a frequency range from $23 \mathrm{GHz}$ to $40 \mathrm{GHz}$ monitoring low wave number turbulence. The antenna array consists of one launcher surrounded by 4 receiver 
antennae (denoted as B,C,D and E). The sight lines of all antennae are discribed by the normal vectors of the antenna mouth and aim at a common focus point inside the plasma vessel at $R, z=(6.0 \mathrm{~m},-0.104 \mathrm{~m})$. Depending on the magnetic configuration this position corresponds for the standard configuration to an effective radius of $r_{\text {eff }}=0.122 \mathrm{~m}$ and for the high iota configuration to $r_{\text {eff }}=0.088 \mathrm{~m}$. The system monitors density fluctuations propagating on flux surfaces which corresponds to a certain density fulfilling the condition $2 \pi f_{r e f}=\omega_{p l}$, where $f_{r e f}$ is the probing frequency of the reflectometer and $\omega_{p l}$ is the plasma frequency. The data of the six different receiver combinations is cross correlated to determine the propagation time $(\Delta t)$ of the density fluctuation as function of the separation between their reflection points [7]. From the distance between the reflection points $(\Delta s)$ the turbulence rotation is estimated for each combination as $v_{\perp}=\Delta s / \Delta t$. To reduce the error bar in the measurement a linear regression consisting of all combinations is performed. In general the measured $v_{\perp}$ is expressed as:

$$
v_{\perp}=v_{E \times B}+v_{\text {phase }}
$$

where $v_{E \times B}$ denotes the $E \times B$ velocity and $v_{\text {phase }}$ an additional phase velocity. With the knowledge of $B_{\phi}$ and neglecting $v_{p h a s e}, E_{r}$ can be calculated as $E_{r}=v_{E \times B} B_{\phi}$. In addition the obtained $v_{\perp}$ has to be corrected by a factor $\gamma=\left(1-\left(\tau_{A C F} / \tau_{d c}\right)^{2}\right)$ [8] which takes into account the convection and distortion of turbulent eddies. Here $\tau_{A C F}$ denotes the mean width at $1 / e$ level of the autocorrelation function from all antenna and $\tau_{d c}$ denotes the decorrelation time of the turbulence defined as the $1 / e$-level of the envelope of all six cross correlation functions. Moreover, the coherence spectrum of all combinations is measured. It yields information on the spectral distribution of the turbulence as function of the radius and allows the decomposition in different components as broad band turbulence and low frequency turbulence as decribed in ref [9].

With the knowledge of the density profile from Thomson scattering diagnostic (TS) [10] the radial reflection position in the plasma is determined. Due to the stellarator geometry the plasma cross sections at the toroidal position of the PCR and the TS are different and a mapping of the measurement positions to an effective radius $\left(r_{e f f}\right)$ is necessary. The shape of the density profile $\left(n_{e}\left(r_{e f f}\right)\right)$ is determined by an exponential fit of the form:

$$
n_{e}\left(r_{e f f}\right)=n_{e 0} \exp \left(-c_{1} r_{\text {eff }}^{2}-c_{2} r_{\text {eff }}^{4}-c_{3} r_{\text {eff }}^{6}\right)
$$

where $c_{1}$ to $c_{3}$ are fit parameters and $n_{e 0}$ is adjusted with the line averaged density measured by interferometer [11]. An example for a typical profile estimation is shown in fig. 1. The density scale length determines the radial resolution of the PCR diagnostic. In the analyzed experiments the radial resolution $(\Delta r)$ is in the range $6 \mathrm{~mm}$ to $12 \mathrm{~mm}$.

In addition to the PCR system a combined probe head is used allowing simultaneously measurement of edge radial profiles as magnetic field, electron temperature and density and floating potential. The triple Langmuir probe with constant biasing consists of four closely packed Langmuir pins, two floating, one for electron collection and the other ion collection on the combined probe head [12]. The probe data is sampled at $1 \mathrm{MHz}$. In contrast to the PCR 
system, it determines $E_{r}$ directly from the floating potential and the electron temperature as:

$$
E_{\mathrm{r}}=-\frac{\partial\left(U_{\text {float }}+2.8 \cdot T_{\mathrm{e}}\right)}{\partial r}
$$

Together with the knowledge of $B$ a measurement of the $E \times B$ rotation is possible. The probe head is mounted on the mid plane manipulator (MPM) at W7-X [13] which is toroidally apart from the PCR system by $\Delta \phi=129.6^{\circ}$ and can be plunged several times during one discharge.

\section{Observations}

The flat top phase of program 20171109.52 is used to compare the calculated position of the LCFS with the inversion point from the $v_{\perp}$-profile, which is obtained from a frequency scan in steps of $\Delta f_{r e f}=500 \mathrm{MHz}$. Fig. 2a shows the Poincare plot obtained from VMECcalculations [14] for the standard configuration (EJM) with an extension showing the flux surfaces outside the LCFS. The calculations are based on the coil currents for the ideal configuration and take into account the $m=1$ error field compensation by the trim coils. Furthermore the $\beta=0$ is assumed. The solid red line denotes the LCFS as calculated by VMEC. Outside the LCFS, an island belonging to the $5 / 5$ island chain is visible. Note that the calculation does not take into account the divertor plates which intersect the island. Beside the calculation the dashed line shows the line of sight $(\mathrm{LoS})$ of the PCR launcher. The measured velocities are obtained during the flat top phase of the discharge and denoted by squares. Note, $v_{\perp}$ is scaled in a way that $v_{\perp} \geq 0$ are above and $v_{\perp} \leq 0$ are below the LoS. For the plasma conditions in 20171109.52 the measurement of the PCR system covers a substantial region in the SOL, but, also a small part in the plasmas edge. The transition from negative to positive $v_{\perp}$ is clearly visible and agrees, within the radial resolution of the PCR system of $\Delta r=8 \mathrm{~mm}$, with the position of the LCFS. In fig. $2 b$ the corresponding connection length to the divertor, $L_{c}$, is shown. The steep increase of the connection length at $R=6.20 \mathrm{~m}$ coincides well with the inversion point in the $v_{\perp}$-profile. The $v_{\perp}$-data show a second transition with $v_{\perp} \leq 0$ inside the island, but, with smaller $\left|v_{\perp}\right|$ compared to the data inside the LCFS. This is the remainder of the $5 / 5$ island winding around the confined plasma. Due to the intersection with the upper and lower divertor it is to a large part destroyed and a small bit can survive only. The width of the remainder island is $\approx 0.01 \mathrm{~m}$ and therefore too small to cause a flattening of the density profile.

For the analysis of the reflectometer data in terms of $v_{E \times B}$, the role of the phase velocity $v_{\text {phase }}$ is essential. Therefore the measurement of $E_{r}$ from PCR and probes is compared. Such a comparison has been performed earlier at AUG [15] demonstrating a good agreement between the measurements. However, the $v_{\text {phase }}$ has not been estimated.

For a sufficiently high flat top density, a small overlap exists between radial positions probed by the PCR and the plunge depth of the MPM in W7-X plasmas. During the plunge of the MPM the probe head does not cross the LCFS. Furthermore, the time of the plunge is aligned to the frequency scan of the PCR in a way that the maximum plunge depth correlates to the outermost position probed by the PCR. Radial and spatial resolutions of $E_{r}$ from the probe are much higher than for the PCR-system. For the comparison $E_{r}$ from the probe is averaged for 
the relevant radial steps of the PCR system according to $r_{\text {eff }}-\Delta r / 2 \leq r_{\text {eff }} \leq r_{\text {eff }}+\Delta r / 2$ where $\Delta r$ denotes the radial resolution of the PCR diagnostic. In fig. 3 the $E_{r}$ from probe data is compared with the PCR data showing a good agreement between both diagnostics within the error bar of the measurement. The decreased innermost value of the MPM data suggests that also the transition from positive to negative $E_{r}$ is in agreement for both diagnostics. Especially the transition from positive to negative $E_{r}$ agrees well with the calculated position of the LCFS for the standard configuration. From the difference in $E_{r}$ obtained from probes and PCR outside the LCFS an estimate of $v_{\text {phase }}$ can be deduced as:

$$
v_{\text {phase }}=\frac{\left\langle E_{r}^{M P M}\right\rangle}{B}-\frac{\left\langle E_{r}^{P C R}\right\rangle}{B}
$$

where \langle\rangle stands for radial averaging outside the LCFS. Taking the three outermost MPM values, which are not to close to the LCFS, an estimate for the phase velocity using equ. 4 yields $v_{\text {phase }} \leq 0.4 \mathrm{~km} \mathrm{~s}^{-1}$.

In general, the phase velocity allows to identify the underlying instability driving the turbulence. A similar estimate has been obtained for the plasma edge of AUG plasmas [16]. The determination of the $v_{\text {phase }}$ allows to conclude on the underlying instability. For the AUG case GEMR simulations [17] indicate that at low wave numbers resistive ballooning turbulence are a good candidate to explain the small $v_{\text {phase }}$. This could be similar for the W7$\mathrm{X}$ plasmas, keeping in mind that the relevant parameters $L_{n}=7 \mathrm{~cm}$ and $\rho_{s}=0.17 \mathrm{~cm}$ are in the same order.

Due to the different currents in the planar and non planar coils, different magnetic configuration can be selected. Fig. 4 shows the effect of the standard (EJM) and the high iota (FTM) configuration on the SOL for two plasmas with similar values in $P_{E C H}, W_{d i a}$ and $n_{e}$ for one frequency scan of the PCR. Note the LCFS for FTM-configuration is located $\approx 0.03 \mathrm{~m}$ deeper as for the EJM configuration. VMEC calculations and PCR measurement are in both cases in agreement. Also from the $L_{c}$ calculations the transition from the SOL to the confined plasma is found at this position. Beside this shift, the SOL width for FTM$(0.02 \mathrm{~m})$ is much smaller than for EJM-configuration $(0.05 \mathrm{~m})$ and mainly due to lack of the large 5/5 island chain. The values of $v_{\perp}$ at the LCFS are larger in FTM than in EJM. In the far SOL the values drop to $v_{\perp} \approx 1 \mathrm{~km} \mathrm{~s}^{-1}$ yielding a significant velocity shear across the SOL. In the EJM case a remainder 5/5-island is visible in the broad SOL region. In contrast, the 5/4-island in the SOL of FTM configuration has a width along the sight line of the PCR diagnistic of $\leq 5 \mathrm{~mm}$ and cannot be detected by the diagnostic.

Interesting is the effect of the toroidal plasma current $\left(I_{p}\right)$ on the size and position of the 5/5-island and the $E_{r}$ and $v_{\perp}$ profiles, respectively. Two components contribute to $I_{p}$, (i) the bootstrap current and the (ii) parallel Pfirsch-Schlüter current compensating the poloidal current. The plasma current in EJM configuration is evolving while main plasma parameters as $P_{E C H}, W_{d i a}, n_{e}$ are kept constant (fig. 5a,b), it has an influence on the remainder island as shown in fig. $5 \mathrm{~d}$. Here the delay time $(\Delta t)$ is shown for one antenna combination and 4 subsequent scans of the reflectometer. Whereas the $1^{\text {st }}$ scan is performed during the ramp up of the density, scans 2-4 are performed during the flat top phase at higher density where the radial interval of the the reflectometer measures in the SOL $\left(0.50 \mathrm{~m} \leq r_{\text {eff }} \leq 0.57 \mathrm{~m}\right)$. For 
the $2^{\text {nd }}$ scan a clear indication of a remainder island is observed and marked by the dashed box at $t=1$ second where $\Delta t<0$. The $3^{r d}$ scan at higher $I_{p}$ shows still the remainder island (dashed box at $t=1.8$ second), but, at a slightly deeper radial position and less pronounced. In the last scan the remainder island has disappeared completely. The expected position is denoted by the dashed box at $t=2.5$ second. Such an effect is observed for a lot of plasmas where $I_{p} \leq 2 \mathrm{kA}$. At higher $I_{p}$ the remainder island seems to disappears in the PCR measurements. A poloidal movement of the island can be excluded since the control coils are not powered in this discharge. Another explanation can be derived from the properties of the O-mode reflection condition which does not allow to measure in regions with flat or even hollow density profiles. From simulations of the connection length of field lines to the divertor, an increase in the size of the remainder $5 / 5$-island is observed due to an increased $I_{p}$ [18]. However, an increase of the size of the remnant island is an adiabatic process which causes a profile flattening. In this case the island is no longer visible in the measurement of the PCR. Also in the density profile the island is not visible because (i) the laser beam of the Thomson scattering diagnostic touches at the outboard side only the edge of the island and (ii) the distance between the scattering volumes is larger than the island width (see fig. 1). A comparison of similar programs with respect to $n_{e}, P_{E C H}$ and $W_{d i a}$ is performed consisting of program 20171109.58 with $I_{p}=1.2 \mathrm{kA}$ and 20171115.11 with $I_{p}=5.1 \mathrm{kA}$ in fig. 6 . It shows $v_{\perp}$ profiles as function of $r_{e f f}$ and the LCFS as estimated from the connection length $\left(L_{c}\right)$ calculation for both plasmas at the PCR position, taking the LCFS at the radial position where $L_{c}$ has dropped by $50 \%$ with respect to the value inside the plasma edge. This calculation is performed for $\beta=0 \%$, but, VMEC calculation for the appropriate plasma $\beta$ of $\approx 0.4 \%$ result in an outward shifted LCFS. To achieve consistency between the measured data and the calculated LCFS a shift of $20 \mathrm{~mm}$ is added to the position of the LCFS from the connection length estimation. For $I_{p}=5.1 \mathrm{kA}$ the LCFS, as estimaed from $L_{c}$, is found $10 \mathrm{~mm}$ more inward, compared to the case with $I_{p}=1.2 \mathrm{kA}$. This relative change is clearly reproduced in the $v_{\perp}$-profiles. Furthermore, an increase of $v_{\perp}$ at the LCFS from $v_{\perp}=4 \mathrm{~km} \mathrm{~s}^{-1}$ to and $v_{\perp}=8.5 \mathrm{~km} \mathrm{~s}^{-1}$ is observed, whereas the values outside the outer separatrix of the $5 / 5$ island are equal. Beside the shift of the LCFS, the width of the $5 / 5$ island increases slightly for $I_{p}=5.1 \mathrm{kA}$. This causes a $n_{e}$-profile flattening. Note that the PCR sight line is about $0.1 \mathrm{~m}$ below the mid plane which is the symmetry axis of the island (see fig. 2). Therefore, the expected change of the island width at the PCR sight line is less pronounced. For both plasmas it is reasonable to assume that the $r_{\text {eff }}$ for the lowest probing frequency is the same. To achieve this, $v_{\perp}$ data for $r_{e f f} \leq 0.56 \mathrm{~m}$ are outward shifted by $20 \mathrm{~mm}$ yielding a nice agreement of $v_{\perp}$ outside the island separatrix. The formed gap is a consequence of the remainder island. The observations suggest a 2 fold effect of the plasma current on (i) the magnetic topology and (ii) on measured values of $v_{\perp}$ and $E_{r}$ in the SOL.

Finally, $v_{\perp}$ and $E_{r}$ are compared for different heating power levels but similar $n_{e}$ in the FTMconfiguration. For both plasmas a frequency scan is performed for the same time interval. In fig. 7 the effect of low and high power on the $v_{\perp}$ - and $E_{r}$-profiles is shown. Again a good agreement between the calculated LCFS and the inversion point in the measured profiles is observed. No significant difference between the $v_{\perp}$ - and $E_{r}$-profiles for the two power levels 
Table 1. Autocorrelation time, decorrelation time and effective poloidal wave length for the remnant island, SOL and plasma edge in EJM configuration for 20171109.54.

\begin{tabular}{cccc}
\hline & Remnant Island & SOL & Plasma edge \\
\hline$\tau_{A C F}$ & $11.4 \mu \mathrm{s}$ & $6.7 \mu \mathrm{s}$ & $5.7 \mu \mathrm{s}$ \\
$\tau_{d c}$ & $20.9 \mu \mathrm{s}$ & $11.0 \mu \mathrm{s}$ & $13.5 \mu \mathrm{s}$ \\
$\lambda_{e f f}$ & $26 \mathrm{~mm}$ & $30 \mathrm{~mm}$ & $50 \mathrm{~mm}$ \\
\hline
\end{tabular}

is observed. Therefore, the effect of $P_{E C H}$ in the FTM-configuration is marginal.

Beside the studies of the rotation and radial electric field, the spectral properties of the turbulence are investigated, too. As an example the coherence of the antenna combination EC is shown in fig. 8 as function of time, respectively radius $\left(0.53 \mathrm{~m} \leq r_{\text {eff }} \leq 0.59 \mathrm{~m}\right)$ obtained from a scan of the reflectometer frequency. Three different radial regions can be identified in the coherence but also in the $\Delta t$-evolution which is shown as solid line, too. The regions are: (i) Far SOL (outside of the remainder island) where the spectrum extents towards $\pm 100 \mathrm{kHz}$, (ii) the remainder island with a narrow spectrum followed by (iii) the SOL region similar in its properties as the one observed in the far SOL and (iv) the plasma edge where the spectrum becomes broad up to $\approx \pm 200 \mathrm{kHz}$. In between the SOL and the plasma edge the LCFS is located where the spectrum is a bit more narrow again. In the plasma edge a quasi coherent mode is observed [19] at $f=23 \mathrm{kHz}$. The mode is located inside the LCFS. The wave number amounts to $k_{\perp}=2 \pi f_{Q C} / v_{\perp}=0.29 \mathrm{~cm}^{-1}$ with a poloidal wave length of $l_{\perp}=0.2 \mathrm{~m}$. Taking the electron temperature from TS measurements at the position of the mode yields $k_{\perp} \rho_{e}=0.1$, suggesting drift ballooning modes to cause this fluctuation. This is in agreement with the small phase velocity of the turbulence as it was deduced from the comparison of $E_{r}$ between MPM and PCR.

From the auto- and cross-correlation analysis for the remainder island, the SOL and the plasma edge significant changes of the turbulence are observed. In tab. 1 the results are summarized. The auto correlation time is taken from the width at $1 / e$ level. A large $\tau_{A C F}$ indicates that the turbulence is not much perturbed and long living. The effective poloidal correlation length $\lambda_{\text {eff }}$ is determined from evolution of the cross correlation coefficient at $\Delta t=0$ for all combinations [8]. In the remainder island the turbulence is long living and not much disturbed. The SOL region as well as the plasma edge have much smaller $\tau_{A C F}$ (a factor of 1.5 -2) compared to the remainder island. The de-correlation time is reduced by nearly the same factor. This is a consequence of the broad coherence spectrum with a significant amount of high frequency perturbations.

For the analysis of the coherence spectrum from the LCFS inwards, the spectrum in the frequency range $-400 \mathrm{kHz}$ to $400 \mathrm{kHz}$ is decomposed into a dc component at zero frequency, a broad band (BB) turbulence component and a component describing the quasi coherent (QC) mode. Each single component can be described by a single Gaussian shape. For the program 20171109.56 the spectra of one antenna combination (EC) are investigated with respect to the BB-turbulence in the plasma edge. In fig. 9 three spectra are shown at different distances to the LCFS. It can be seen that the contribution of the BB-turbulence increase with increasing 
distance to the LCFS. The QC mode center frequency decreases and the mode becomes much broader. Due to the large velocity shear at the LCFS it is assumed that turbulent eddies are torn apart [20,21]. Monitoring the BB-turbulence as function of the distance from the LCFS allows to estimate the radial correlation length. To describe the radial evolution of the BB turbulence, the spectra are integrated in the range $-300 \mathrm{kHz}$ to $300 \mathrm{kHz}$ and each spectrum is normalized with respect to the innermost one. The contribution of the BB-turbulence is taken as the area below the fitted shape of the BB-turbulence and shown in percent as function of $r_{e f f}$ in fig. 10. For this program the LCFS is located at $r_{e f f}=0.53 \mathrm{~m}$ as calculated from the PCR $v_{\perp}$ profile. At $r_{\text {eff }}=0.51 \mathrm{~m}$ the BB-turbulence accounts for $83 \%$ of the coherence spectrum. With decreasing distance to the LCFS the contribution decreases quickly yielding $38 \%$ at $r_{\text {eff }}=0.526 \mathrm{~m}$. The decrease in the BB-turbulence stems from the velocity shear at the LCFS. From the decay of the BB-turbulence towards the LCFS an estimate of the radial extension $\left(\lambda_{r}\right)$ of the BB-turbulence is possible. It is shown by the solid line in fig. 10 where a Gaussian with $1 / e$ width of $15 \mathrm{~mm}$ is an upper limit for $\lambda_{r}$. The analysis demonstrates that even without a radial correlation measurement $\lambda_{r}$ can be estimated given a localized shearing layer in the radial interval of the measurement.

\section{Summary}

The plasma edge and the SOL region of diverted plasmas in $\mathrm{W} 7-\mathrm{X}$ is investigated by a poloidal correlation reflectometer and a set of Langmuir probes to study radial profiles of $v_{\perp}$ and $E_{r}$. Depending on the plasma parameters the reflectometry is able to detect the inversion point in the $v_{\perp}$ - and $E_{r}$-profiles, respectively. Within the radial resolution of the diagnostic $(\Delta r \leq 10 \mathrm{~mm})$ this radial position corresponds clearly with the calculated LCFS position from VMEC calculations as well as to the increased connection length, assuming $\beta=0 \%$. This allows for low and medium density to monitor the inversion point in the $v_{\perp}$-profile which is a measure for the position of the LCFS.

From the difference turbulence- and $E \times B$ - rotation, the assumption $v_{E \times B} \gg v_{\text {phase }}$ is justified in the plasma edge and SOL. Furthermore the small phase velocity supports resistive ballooning modes as the dominating instability at the plasma edge.

For two magnetic configurations the SOL width is measured. In the high iota case the SOL is narrow, compared to the configuration which exhibits a $5 / 5$ island chain in the SOL. In the latter configuration a remainder island has been observed which is subject to changes of

$I_{p}$. With increasing plasma current the remainder island becomes broader and an evidence for a flattening of the electron density is found. In this cases an increase of plasma rotation is detected when the plasma current increases.

The analysis of the coherence spectrum shows that the turbulence in the remainder island is weak and the spectrum is narrow, whereas in the SOL and the plasma edge the turbulence is much stronger with a broader spectrum. Due to the large shear at the last closed flux surface the broad band turbulence disappears. In the plasma edge the broad band turbulence becomes dominant again and reaches its full power $20 \mathrm{~mm}$ away from the last closed flux surface and inside the plasma edge. It allows to estimate the radial extent of the broad band turbulence of 
$\lambda_{r} \approx 15 \mathrm{~mm}$ for these plasmas.

\section{Acknowledgments}

This work has been carried out within the framework of the EUROfusion Consortium and has received funding from the Euratom Research and Training Programme 2014-2018 under Grant Agreement No. 633053. The views and opinions expressed herein do not necessarily reflect those of the European Commission.

\section{References}

[1] H. S. Bosch, V. Erckmann, R. W. T. Konig, F. Schauer, R. J. Stadler, and A. Werner. Construction of wendelstein 7-x - engineering a steady-state stellarator. IEEE Transactions on Plasma Science, 38(3):265-273, March 2010.

[2] H.-S. Bosch, R.C. Wolf, T. Andreeva, J. Baldzuhn, and D. Birus et al . Technical challenges in the construction of the steady-state stellarator wendelstein 7-x. Nuclear Fusion, 53(12):126001, 2013.

[3] Y. Feng, F. Sardei, P. Grigull, K. McCormick, J. Kisslinger, and D. Reiter. Physics of island divertors as highlighted by the example of w7-as. Nuclear Fusion, 46(8):807, 2006.

[4] A. Dinklage, C. D. Beidler, P. Helander, G. Fuchert, H. Maassberg, et al. Magnetic configuration effects on the Wendelstein 7-X stellarator. NATURE PHYSICS, 14(8):855+, AUG 2018.

[5] A. Krämer-Flecken, T. Windisch, W. Behr, G. Czymek, and P. Drews et al . Investigation of turbulence rotation in limiter plasmas at $\mathrm{w} 7 \mathrm{x}$ with newly installed poloidal correlation reflectometer. Nuclear Fusion, 57(6):066023, 2017.

[6] T Windisch, A Krämer-Flecken, JL Velasco, A Könies, and C Nührenberg et al . Poloidal correlation reflectometry at w-x: radial electric field and coherent fluctuations. Plasma Physics and Controlled Fusion, 59(10):105002, 2017.

[7] A. Krämer-Flecken, S. Soldatov, B. Vowinkel, and P. Müller. Rev. Sci. Instrum., 81:113502, 2010.

[8] D Prisiazhniuk, A Krämer-Flecken, G D Conway, T Happel, A Lebschy, P Manz, V Nikolaeva, U Stroth, and the ASDEX Upgrade Team. Magnetic field pitch angle and perpendicular velocity measurements from multi-point time-delay estimation of poloidal correlation reflectometry. Plasma Physics and Controlled Fusion, 59(2):025013, 2017.

[9] V.A. Vershkov V.F. Andreev, A.A. Borschegovskiy, V.V. Chistyakov, M.M. Dremin, L.G. Eliseev, et al. Nucl. Fusion, 51:094019, 2011.

[10] E. Pasch, M. N. A. Beurskens, S. A. Bozhenkov, G. Fuchert, J. Knauer, and R. C. Wolf. The thomson scattering system at wendelstein 7-x. Review of Scientific Instruments, 87(11):11E729, 2016.

[11] J. Knauer, P. Kornejew, H. Trimino Mora, M. Hirsch, and A. Werner et al . A new dispersion interferometer for the stellarator wendelstein 7-x. page P4.017. 43rd European Physical Society Conf. on Plasma Physics (Leuven, Belgium), 2016.

[12] P. Drews, Y. Liang, S. Liu, A. Krämer-Flecken, and O. Neubauer et al . Measurement of the plasma edge profiles using the combined probe on w7-x. Nuclear Fusion, 57(12):126020, 2017.

[13] D. Nicolai, V. Borsuk, P. Drews, O. Grulke, and K.P. Hollfeld et al . A multi-purpose manipulator system for w7-x as user facility for plasma edge investigation. Fusion Engineering and Design, 123:960 - 964, 2017. Proceedings of the 29th Symposium on Fusion Technology (SOFT-29) Prague, Czech Republic, September 5-9, 2016.

[14] J. Geiger. private communication. 2018.

[15] H.W. Müller, J. Adamek, R. Cavazzana, G.D. Conway, C. Fuchs, et al. Latest investigations on fluctuations, elm filaments and turbulent transport in the sol of asdex upgrade. Nuclear Fusion, 51(7):073023, 2011.

[16] D Prisiazhnuik, P Manz, G.D. Conway, T Happel, a Krämer-Flecken, et al. Measurement of the turbulent phase velocity in the 1-mode edge of asdex upgrade and comparison with gemr simulations. pages 
P4.173/1-4. 44rd EPS Conference on Contr. Fusion and Plasma Phys., Belfast, Northern Ireland, 26-30 June 2017, 2017.

[17] P. Manz, D. Prisiazhniuk, T. Happel, S. Freethy, K. Hallatschek, B. D. Scott, U. Stroth, and the ASDEX Upgrade Team. On the phase velocity in between weak and strong plasma edge turbulence. Plasma Physics and Controlled Fusion, 60(8):085002, 2018.

[18] Y. Gao, M. Jakubowski, J. Geiger, M. Endler, and P. Drewelow andet al . Effects of toroidal plasma currents on the strike-line movements on w7-x. 45rd European Physical Society Conf. on Plasma Physics (Prague, Czech Republic), 2018.

[19] X. Han, A. Krämer-Flecken, T. Windisch, S. Liu, and Y. Liang et al . Experimental characterization of a quasi-coherent turbulence structure in the edge plasmas in w7-x. 45rd European Physical Society Conf. on Plasma Physics (Prague, Czech Republic), 2018.

[20] G Van Oost, J Admek, V Antoni, P Balan, J A Boedo, et al. Turbulent transport reduction by e b velocity shear during edge plasma biasing: recent experimental results. Plasma Physics and Controlled Fusion, 45(5):621, 2003.

[21] B. Nold, P. Manz, T. T. Ribeiro, G. Fuchert, G. Birkenmeier, H. W. Mller, M. Ramisch, B. D. Scott, and U. Stroth. Turbulent transport across shear layers in magnetically confined plasmas. Physics of Plasmas, 21(10):102304, 2014. 


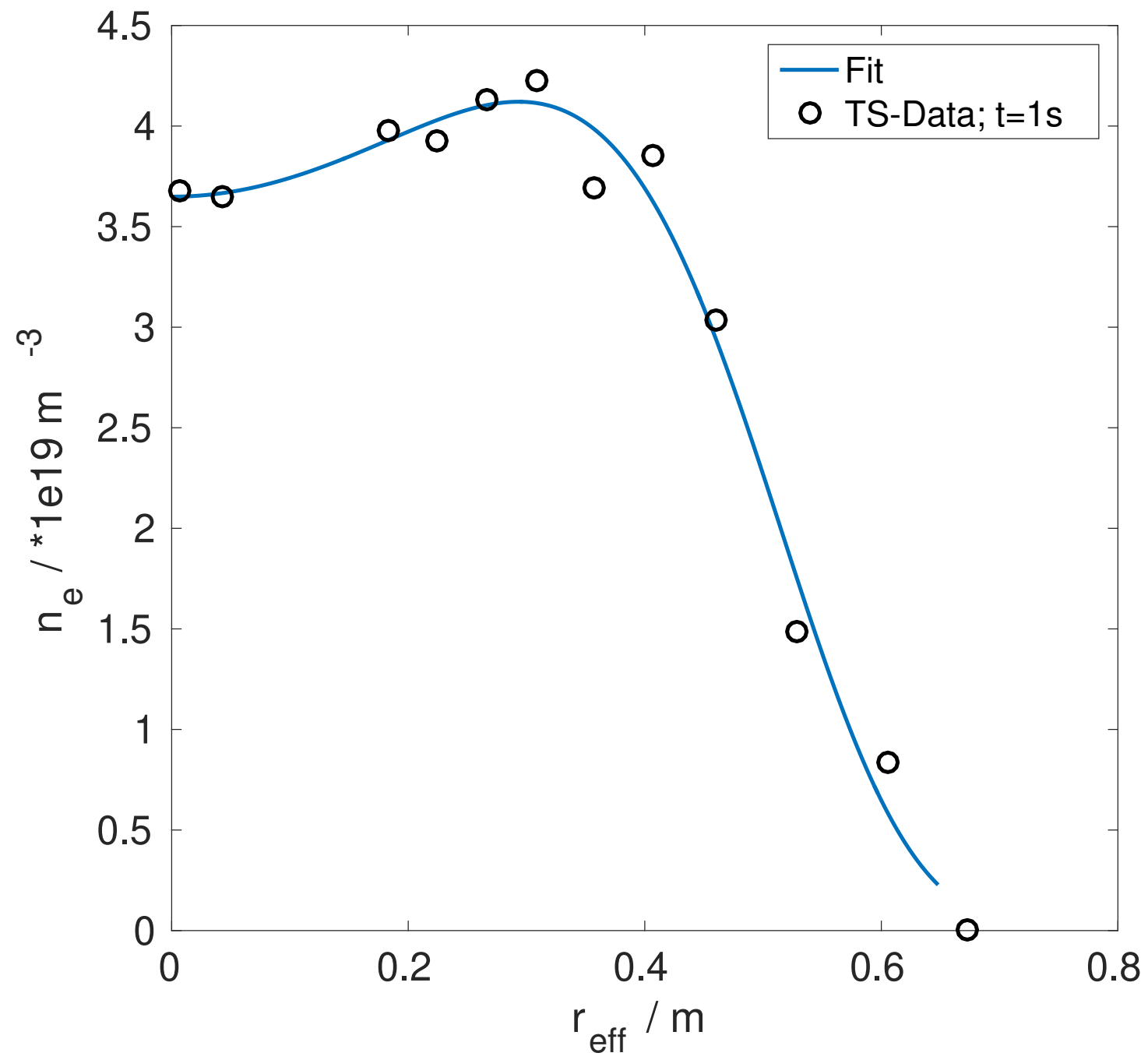

Figure 1. Estimated density profile using equ. [1] for 20171108.40 at $t=1 \mathrm{~s}$. 


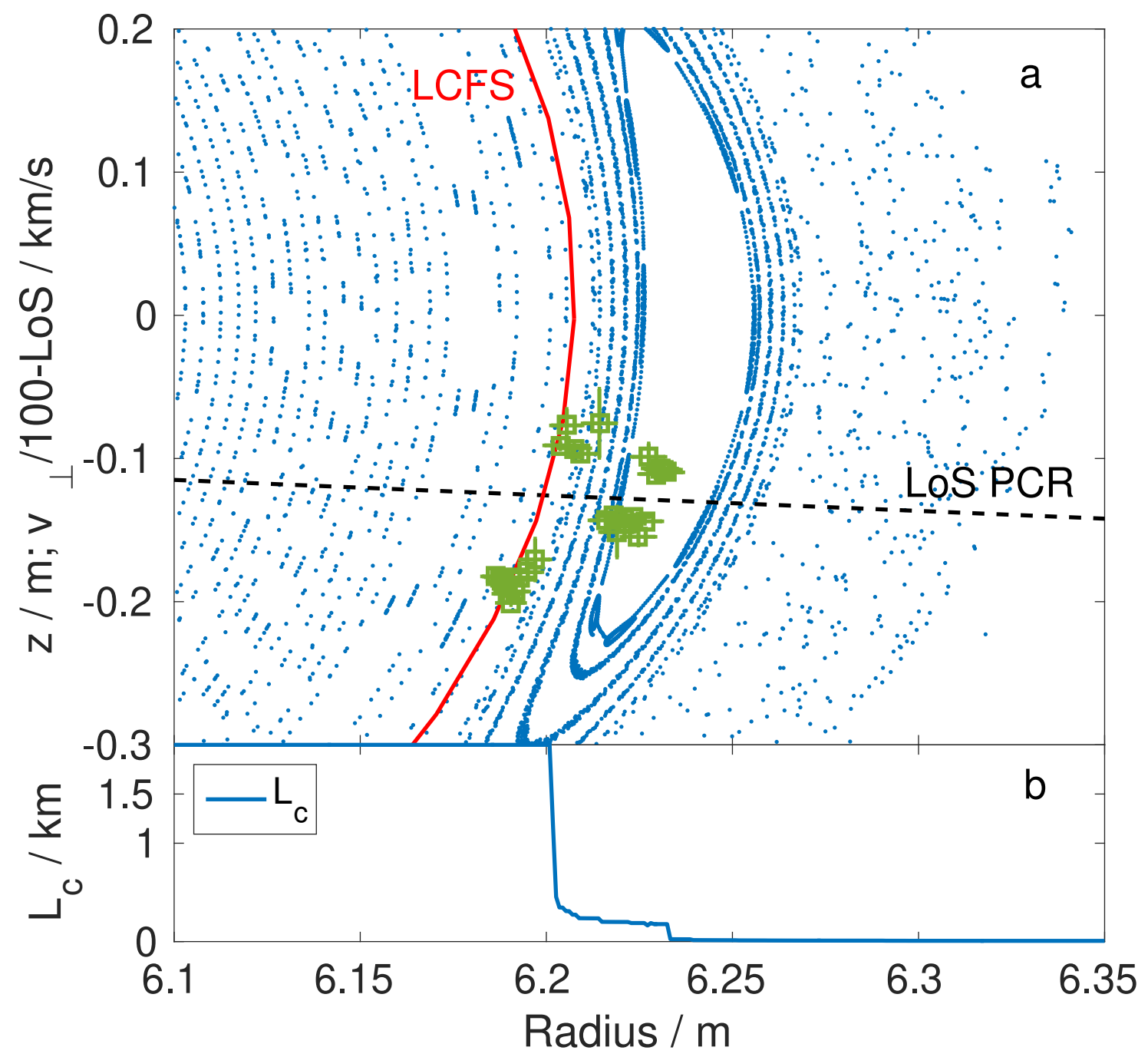

Figure 2. Poincaré calculations for 20171109.52 including the LCFS. Along the LoS the measured $v_{\perp}$ is shown for the time range $0.79 \mathrm{~s} \leq t \leq 1.53 \mathrm{~s}$. For the velocities the LoS fulfils the condition $v_{\perp}=0 \mathrm{~km} \mathrm{~s}^{-1}$. 


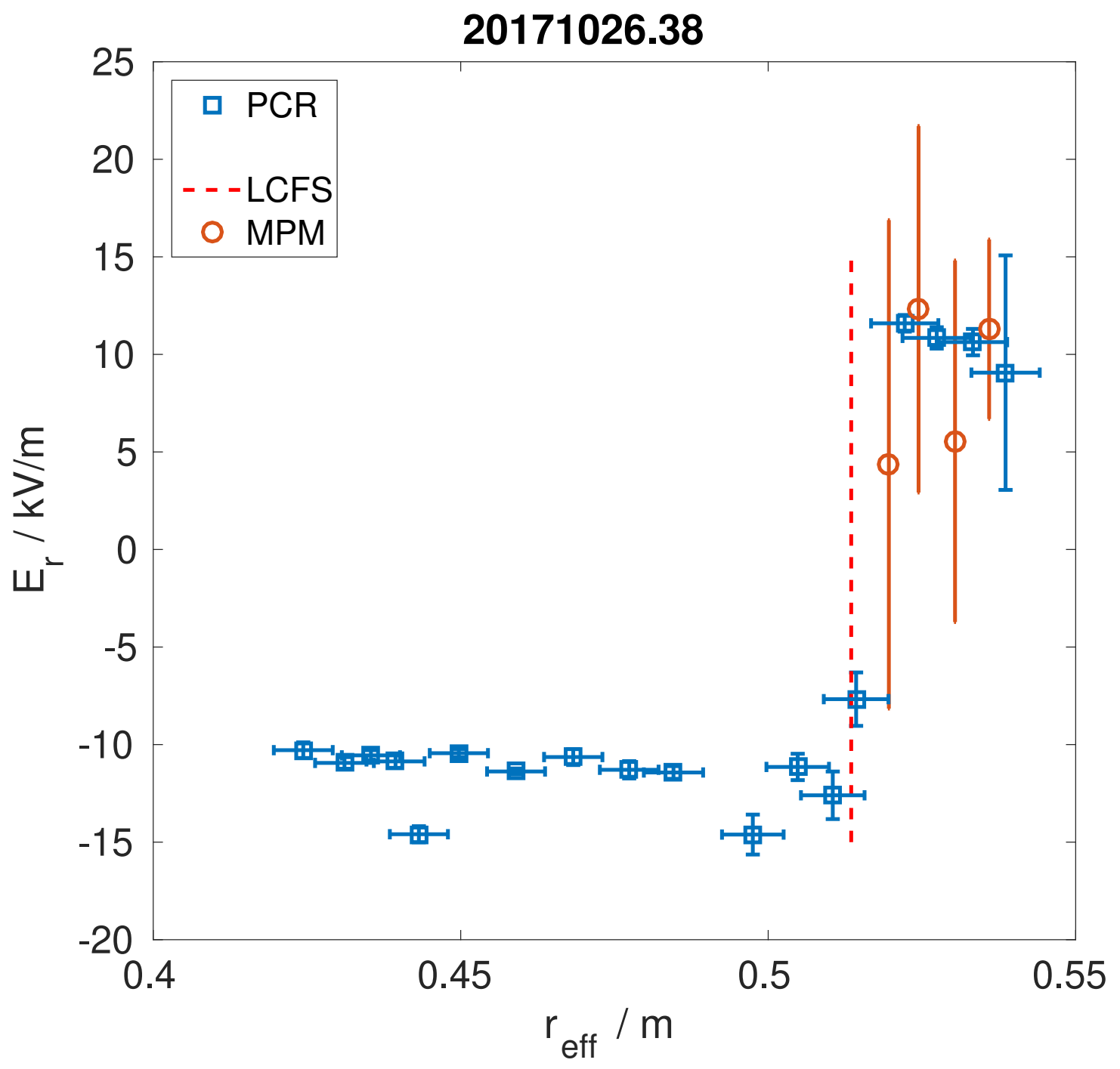

Figure 3. Comparison of $E_{r}$ as calculated from the combined probe head on the MPM and the PCR system. The first one calculates $E_{r}$ from $U_{\text {float }}$ whereas $E_{r}$ from PCR is estimated from $v_{\perp}$. The agreement between both methods suggest a negligible $v_{\text {phase. }}$ The dashed line denotes the LCFS for this discharge 


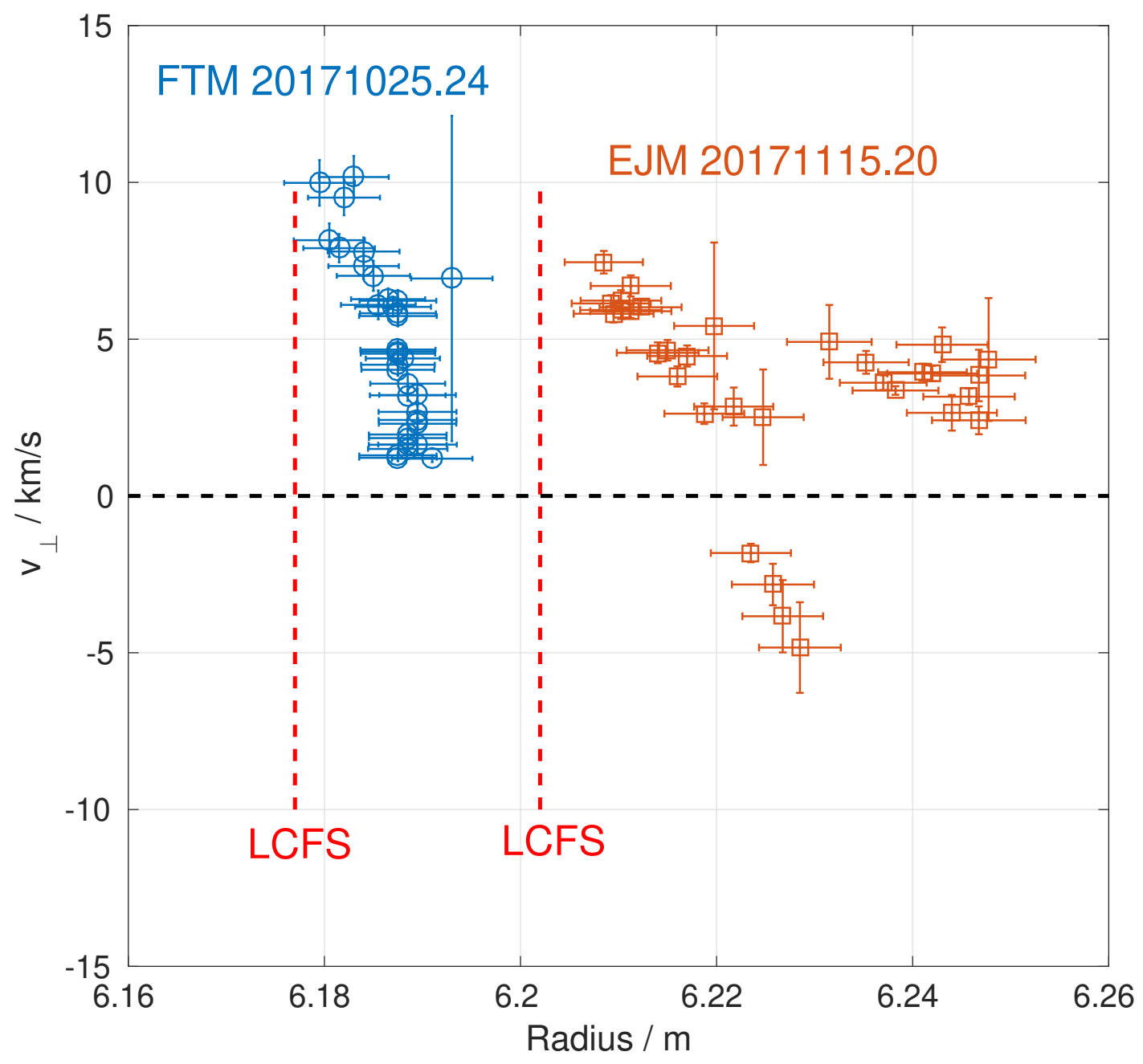

Figure 4. Comparison of FTM and EJM configurations for similar plasma parameters. Note the inward shift of the LCFS and the strong velocity shear in the FTM configuration. 


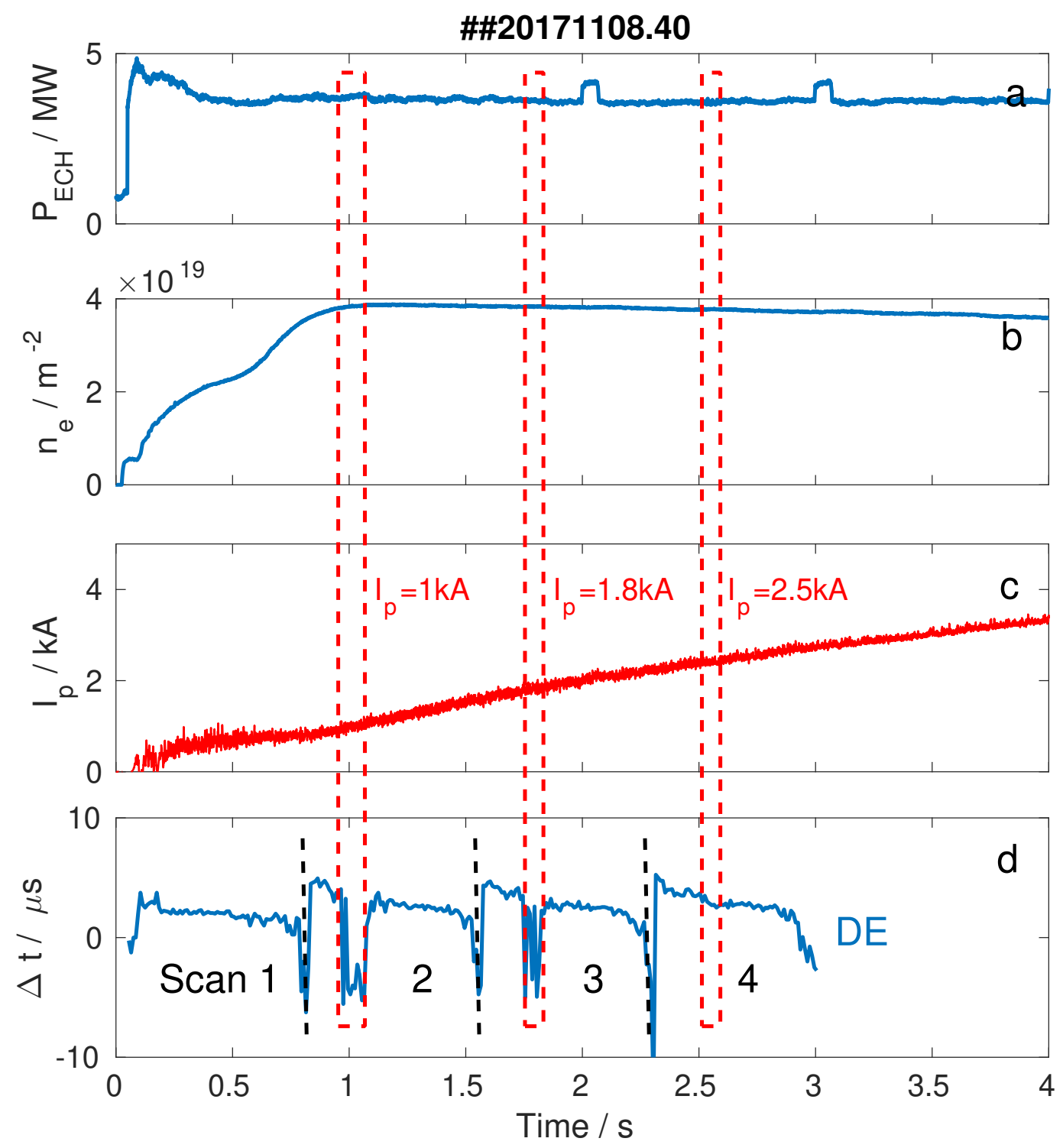

Figure 5. Plasma parameters (a) $P_{E C H}$, (b) line averaged density, (c) $I_{p}$ and $(\boldsymbol{d})$ Propagation time $\Delta t$ from combination $D E$ showing that the remainder island disappear at $I_{p} 2 \mathrm{kA}$. The vertical dashed lines denote the end of the frequency scans. Boxes denotes the time intervals where the remainder island is expected. 


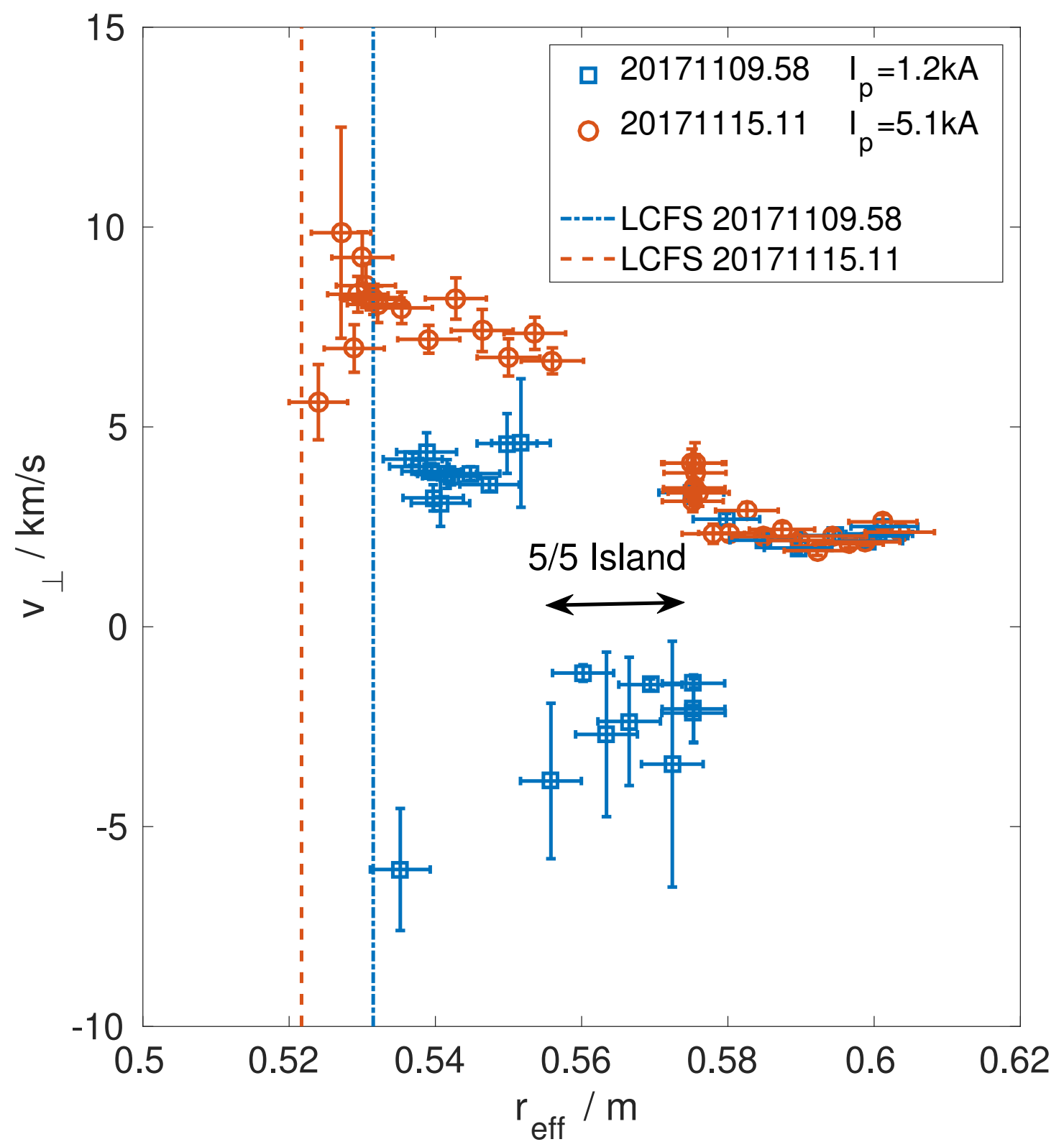

Figure 6. Comparison of $v_{\perp}$ for two similar programs. The main difference is the increase in $I_{p}$ by a factor 4 causing an increase in the $v_{\perp}$ by a nearly a factor 2 between LCFS and the island. Outside the island the $v_{\perp}$ profile are equal. Also the relative shift of the LCFS is agreement with the experimental data. 


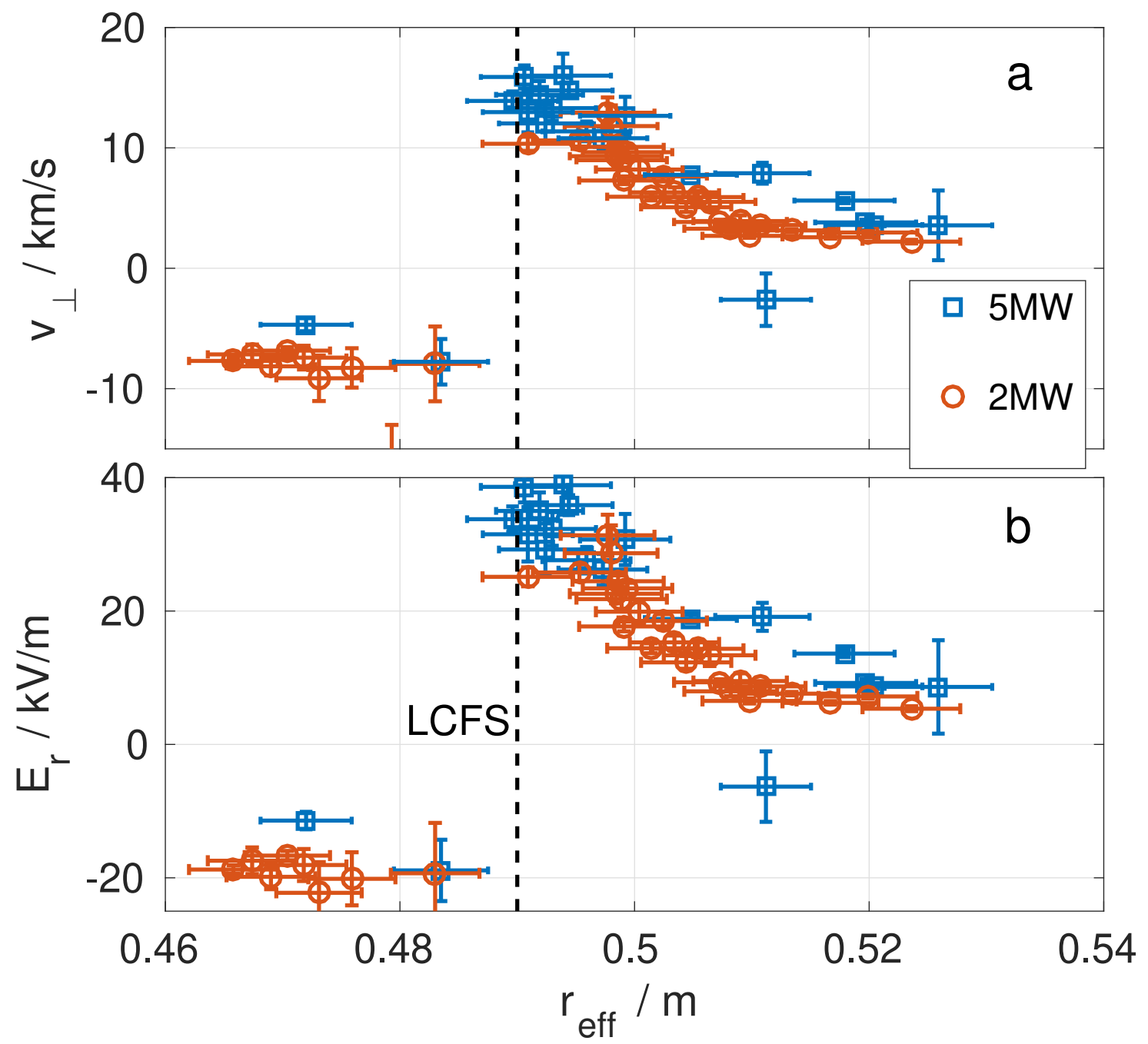

Figure 7. (a) $v_{\perp}$ - and (b) $E_{r}$-profile for different $P_{E C H}$ for similar programs 20171025.16 and 20171025.21. The dashed denotes the position of the LCFS.The time interval of the frequency scan is $0.30 \mathrm{~s} \leq t \leq 0.89 \mathrm{~s}$. 


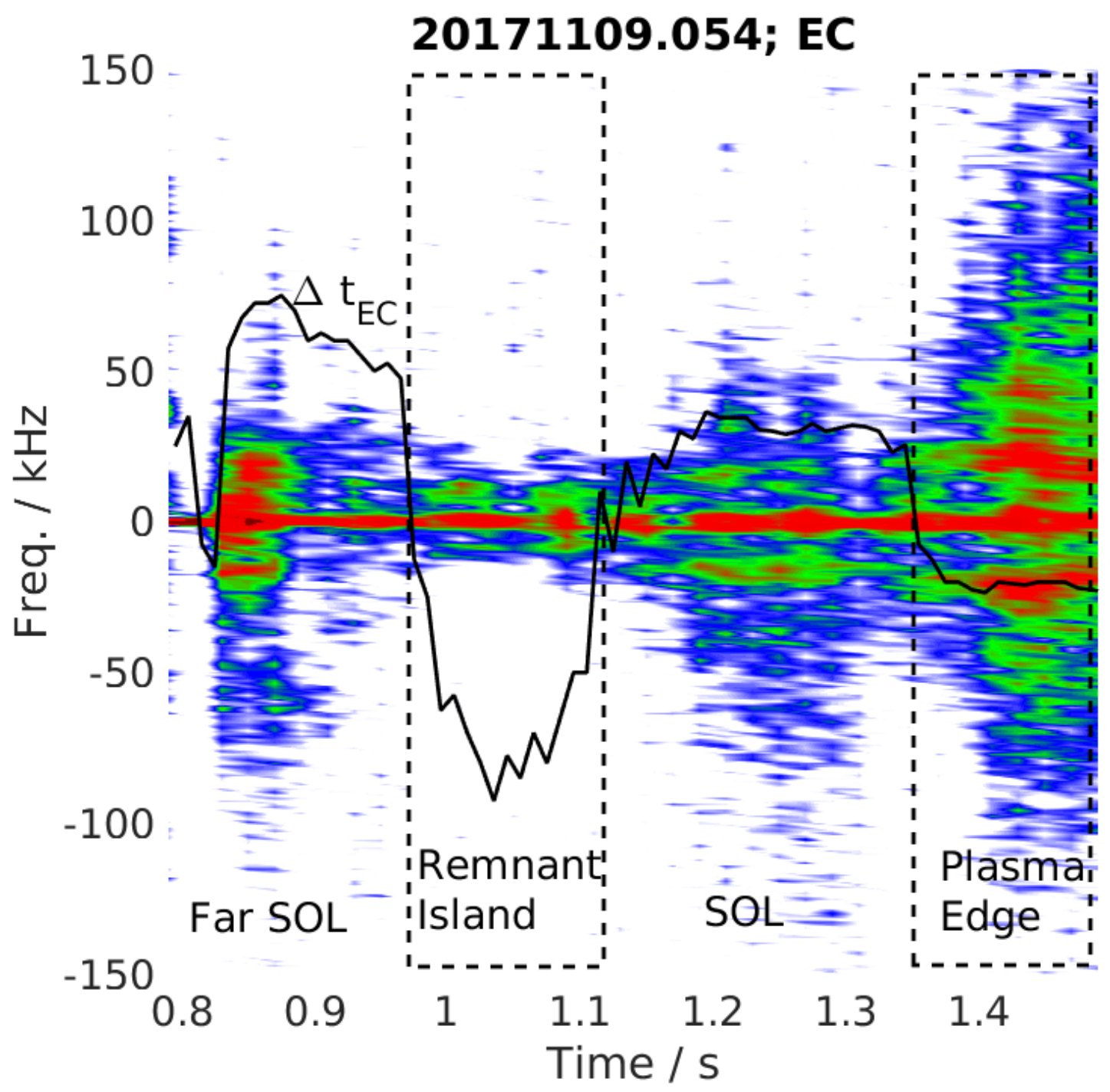

Figure 8. Coherence spectrum for combination EC together with the $\Delta t$ evolution. W7-X is operated in standard combination. From the spectrum four different region can be identified. At the plasma edge a quasi coherent mode is observed. 

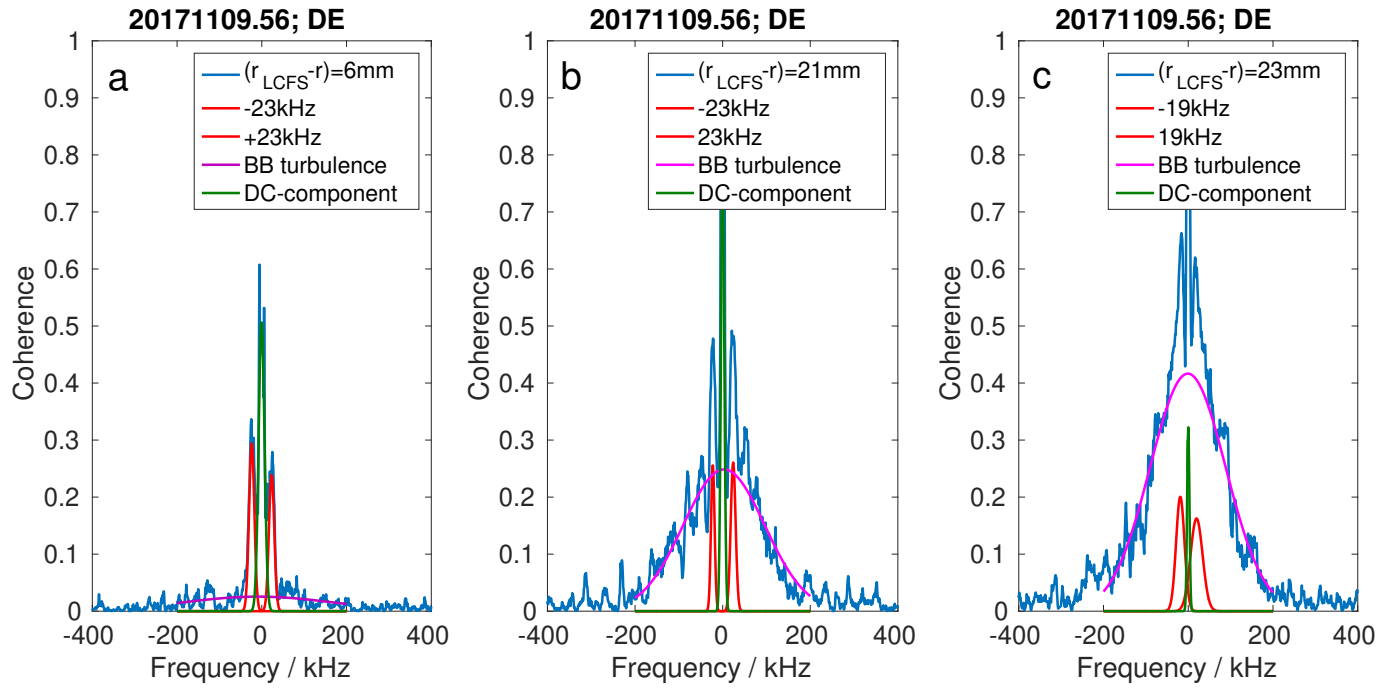

Figure 9. Coherence spectra obtained from combination $D E$ for different distances (increasing distance from (a) to (c) ) with respect to the LCFS. Each spectrum is superposition of BBturbulence, QC-mode at $23 \mathrm{kHz}$ and $19 \mathrm{kHz}$ and a DC component. 


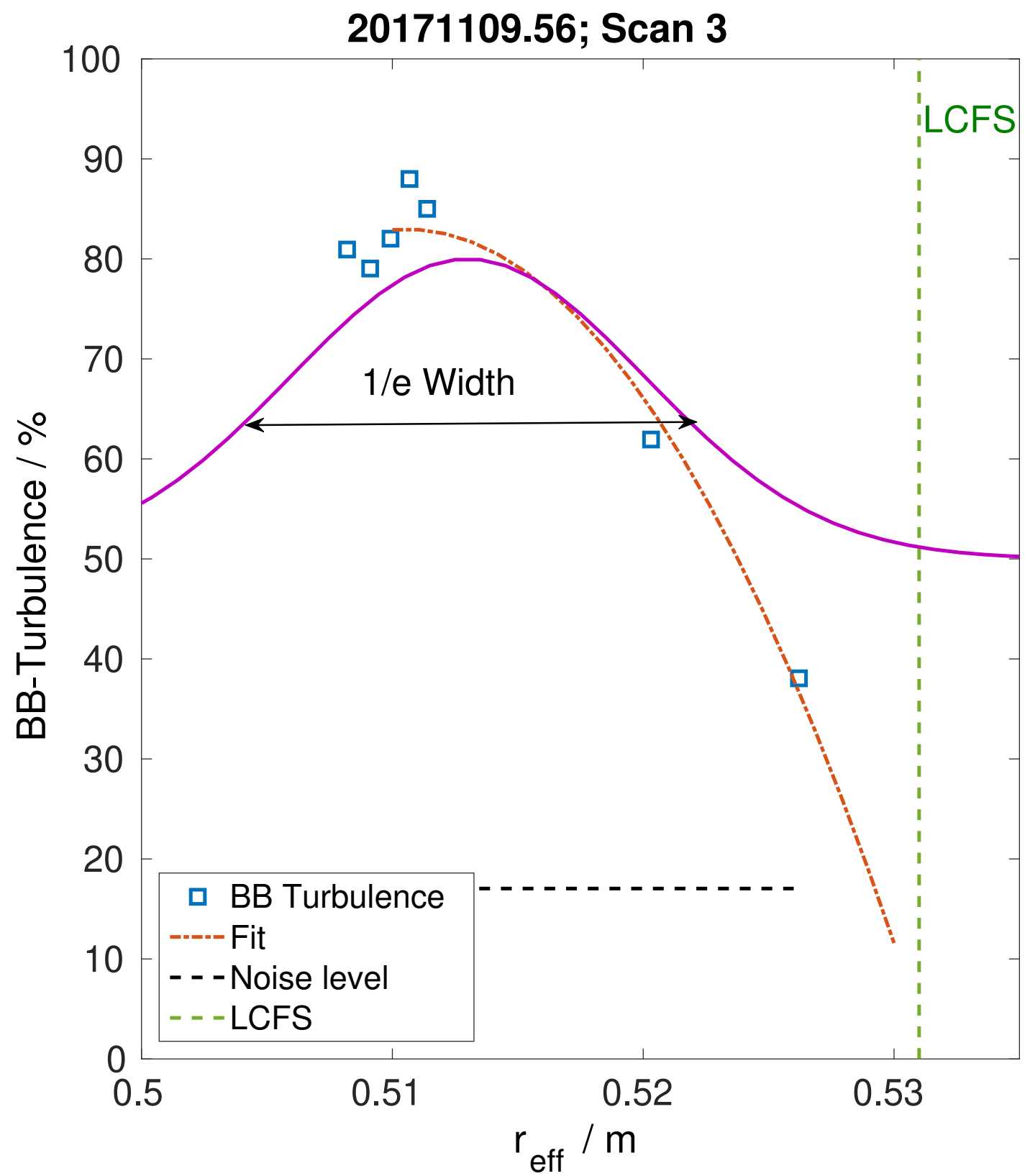

Figure 10. decrease of the BB-turbulence $(\square)$ as function of $r_{\text {ef } f}$. Dashed vertical line denotes the LCFS. The horizontal line is noise level in the estimation of the BB-turbulence. The solid line denotes a Gaussian with $\lambda_{r}=15 \mathrm{~mm}$. The dash dotted line describes the decrease of the $B B$-power towards the LCFS. 